\title{
The Novel Prostate Cancer Antigen 3 (PCA3) Biomarker
}

\author{
Andreas Bourdoumis, Athanasios G. Papatsoris, Michael Chrisofos, Eleni Efstathiou, Andreas \\ Skolarikos, Charalambos Deliveliotis
}

\author{
University Department of Urology (AB, AGP, MC, AS, CD), Sismanoglio Hospital, Athens, Greece \\ and University Department of Clinical Therapeutics (EE), Alexandra Hospital, Athens, Greece
}

\begin{abstract}
PCA3 is a prostate specific, nonprotein coding RNA that is significantly over expressed in prostate cancer, without any correlation to prostatic volume and/or other prostatic diseases (e.g. prostatitis). It can now easily be measured in urine with a novel transcription-mediated amplification based test. Quantification of PCA3 mRNA levels can predict the outcome of prostatic biopsies with a higher specificity rate in comparison to PSA. Several studies have demonstrated that PCA3 can be used as a prognostic marker of prostate cancer, especially in conjunction with other predictive markers. Novel PCA3-based nomograms have already been introduced into clinical practice. PCA3 test may be of valuable help in several PSA quandary situations such as negative prostatic biopsies, concomitant prostatic diseases, and active surveillance. Results from relevant clinical studies, comparative with PSA, are warranted in order to confirm the perspective of PCA3 to substitute PSA.
\end{abstract}

Key words: prostatic neoplasms; biopsy; PCA3; PSA; tumor marker

Int Braz J Urol. 2010; 36: 665-9

\section{INTRODUCTION}

Since its approval by the US Federal Drug Administration in 1986, prostate-specific antigen (PSA) has been employed worldwide to diagnose and monitor men with prostate cancer (PCa) (1). Unfortunately, PSA bears a low positive predictive value, resulting in a significant proportion of negative biopsies, often leading to repeat PSA measurements and biopsies. In men with serum PSA between 3-10 ng/mL (grey zone) the negative biopsy rate is approximately $60-75 \%$ (1). Moreover, PSA-based opportunistic screening has led to an increase in $\mathrm{PCa}$ diagnosis and over treatment due to the high incidence of clinically insignificant PCa (2). Furthermore, men that undergo a PSA test and a consequent biopsy experience anxiety over the result and the outcome (3). Therefore, there is a need for PCa biomarkers with a better specificity to enter clinical practice. Such a perspective biomarker is prostate cancer antigen 3 (PCA3).

\section{WHAT IS PCA3?}

Around 1995, PCA3 was identified in collaborative research effort by Johns Hopkins Hospital, Baltimore and the Radboud University, Nijmegen, Netherlands (4). Initially, it was called Differential Display clone 3 (DD3) as differential display analysis was used to compare mRNA expression patterns of normal versus cancerous prostatic tissue (5). PCA3 is a segment of non-coding messenger ribonucleic acid 
(mRNA) from chromosome 9q21-22 (5). The multiplicity of stop codons across the three reading frames of PCA 3 and the lack of an extended open reading frame indicates that PCA3 functions as a non-coding RNA without encoding a protein (6). Recently, a new genomic structure of PCA3 as well as new flanking and overlapping gene transcripts (e.g. PCA3-TS4 isoform) have been identified (7).

PCA3 is over expressed by more than $95 \%$ of all prostate cancers tested (5). It can determine benign from cancerous prostate cells with an accuracy approaching $100 \%$ (8). Furthermore, no PCA3 transcripts have been detected in extraprostatic tissues (benign and cancerous), demonstrating that PCA3 is the most specific PCa biomarker identified to date (8). Unlike serum PSA, PCA3 is not affected by age, prostate volume or other prostatic diseases (e.g. prostatitis) $(5,7)$.

\section{PCA3 TEST}

PCA3 is measured in urine samples collected after an attentive digital rectal examination (DRE) of the prostate in order for cells to exfoliate in urine. A DRE of three (more strokes share equivalent results) strokes per lobe is performed by applying firm pressure (surface is depressed by approximately $1 \mathrm{~cm}$ ) from the base to apex and from the lateral to the median line for each lobe (9). Without DRE the PCA3 test provides valid results in only approximately $80 \%$ of cases $(5,7)$.

The PCA3 test requires collection of the first 20-30 mL of voided urine after DRE. Approximately $2 \mathrm{~mL}$ of the urine sample is placed into a transport tube with lysis buffer that contains ribonuclease inhibitors (mRNA is destroyed within 20 minutes in the absence of the inhibitors) (8). The transport tube is sent overnight to a specialized laboratory at room temperature but it can also be stored frozen. Some authors use urinary sediments instead of urine samples in order to examine all the cells and cell fragments (10). However, due to simplified procedures whole urinary samples are preferred (10).

Three successive generations of the PCA3 test have been presented, leading to a commercially available kit known as the ProgensaTM PCA3 assay (Gen-Probe, San Diego, CA, USA) (11). Fortunately, this novel fast and easy transcription-mediated amplification (TMA)-based test has replaced the older intensive and time-consuming reverse-transcriptase polymerase chain reaction test (5). The TMA-based PCA3 assay consists of the following steps: i) specific isolation of the RNA of interest by using coated magnetic beds, ii) amplification, and iii) detection and quantification of the isolated RNA (11). Informative rates (percentage of urine samples yielding accurately quantifiable mRNAs for assay) are $>99 \%$ and the assays have good reproducibility (12).

The PCA3 assay provides a quantitative result from the ratio of the mRNA transcripts of PCA3 to PSA. The quantification of PSA mRNA is required to normalize the total mRNA present in a sample. The PSA mRNA levels in prostate cells released in urine are unrelated to PSA protein levels in blood and are essentially unchanged in prostate cancer (12). The PCA3 score is the ratio of PCA3:PSA mRNAs multiplied by 1,000 (5).

The PCA3 score correlates with the results of a subsequent prostatic biopsy as a high score increases the likelihood of a PCa positive result. It has been demonstrated that men with a PCA 3 score $<5$ had a PCa positive biopsy rate of $14 \%$, while $69 \%$ of men with a PCA3 score $>100$ had PCa upon biopsy (5). A cutoff PCA 3 score of 35 has been adopted because in a prospective study among 570 men undergoing prostate biopsy, this score combined the greatest cancer sensitivity and specificity (54\% and $74 \%$, respectively) (13).

\section{CLINICAL IMPLICATIONS}

PCA3 test may be of valuable help in the following PSA quandary conditions: i) elevated PSA with one or more negative biopsies, ii) normal PSA with family history of prostate cancer, iii) normal PSA with positive biopsy, iv) elevated PSA with prostatitis, v) elevated PSA with precursor lesions of PCa (14), and vi) active surveillance for presumed microfocal disease (15). Potential clinical applications include the detection of local recurrence following radical prostatectomy or radiotherapy and monitoring patients on medication that affects serum PSA (i.e. 5-alpha reductase inhibitors) (5). 
Recently, in a review (11) of clinical studies with 2048 men undergoing a first or repeat biopsy, the area under curve (AUC) for PCA3 was 0.66-0.87, while the AUC for PSA always scored less. In comparison with PSA, the specificity of PCA3 was better (66-89\%), while the sensitivity was less (54-82\%). The positive (48-75\%) and negative (74-90\%) predictive values were better for PCA3. In the cohort of 1125 patients with at least one negative biopsy, PCA3 proved (multivariate analysis) to be an independent predictive factor of repeat positive biopsy.

Can PCA3 be considered a prognostic marker for PCa? The association of PCA3 with prostatectomy tumor volume and other clinical/pathological features was assessed by several authors. In most studies PCA3 was significantly correlated with tumor volume, pT stage and Gleason score (GS) (11). Moreover, PCA3 was significantly higher in patients with clinical significant $\mathrm{PCa}$ (dominant tumor volume $>0.5$ cc and GS $\geq 7$ ) (5). However, recently the predictive value of PCA3 for $\mathrm{PCa}$ aggressiveness as reported in earlier studies was not confirmed in a study among 70 men who underwent radical prostatectomy (2). This could be attributed to the usage of urinary sediments instead of whole urine samples and to the small number of patient's favorable prognostic outcome. The prognostic value of PCA3 needs validation in definitive trials.

PCA3 was identified as a statistically independent and informative novel marker that is capable of increasing the predictive accuracy of multivariate biopsy models (16). Predictive accuracy estimates of biopsy outcome predictions were quantified using the AUC of the receiver operator characteristic analysis in models with and without PCA3. Recently, a PCA3-based nomogram has been presented by Chun et al. (16) that identifies men at risk of harboring $\mathrm{PCa}$ and it assists in deciding whether further evaluation is necessary. When PCA3 is combined with serum PSA and biopsy GS, it increased diagnostic accuracy to $90 \%$ to predict extracapsular extension or clinically low volume $(<0.5 \mathrm{cc})$ tumor (17). Patients with a biopsy GS of 6, low PSA and a low PCA3 could be excellent candidates for active surveillance.

The incorporation of PCA3 data into the Prostate Cancer Prevention Trial (PCPT) risk calcula- tor showed additional benefit (18). PCA3, PSA and DRE data from 521 men undergoing prostate biopsy were included in the original PCPT risk calculator. The AUC for the updated PCPT calculator was 0.7, which was statistically superior to the AUC (0.61) of original PCPT calculator without the PCA3. Furthermore, the sensitivity, positive and negative predictive value were improved with the incorporation of the PCA3.

Lastly, patients with precursor lesions of $\mathrm{PCa}$ (prostatic intraepithelial neoplasia, atypical small acinar proliferation) had a significant higher mean PCA3 score, in comparison with men with negative biopsy results $(11,14)$. Nearly one third of patients with a negative biopsy and a PCA3 score $>35$ had high-grade prostatic intraepithelial neoplasia (19).

\section{EPILOGUE}

PCA3 fulfils many of the requirements for PCa biomarker to be useful in clinical practice: i) an easily accessible biological sample (urine after DRE), ii) a reproducible technique across testing laboratories (aided by an available commercial kit), and iii) good statistical accuracy (substantiated by several teams studying populations of different origins) (11). One efficient way to use PCA3 as a guide to choosing repeat biopsy is to collect a PCA3 sample at the time of prostate biopsy ("reflex PCA3") (20). If the biopsy finding is negative, the PCA3 test can be performed without the patient having to return.

Because PCa is a heterogeneous disease, the use of a panel of biomarkers (e.g. PCA3 and TMPRSS2-ERG gene fusions) can further improve diagnostic accuracy (6). The challenge remains to identify biomarkers that can contribute to identify potentially life-threatening $\mathrm{PCa}$ at a curable stage. PCA3 could be such a potential prognostic marker but results from relevant research are warranted.

\section{CONFLICT OF INTEREST}

None declared. 


\section{REFERENCES}

1. Reed AB, Parekh DJ: Biomarkers for prostate cancer detection. Expert Rev Anticancer Ther. 2010; 10: 10314.

2. Hessels D, van Gils MP, van Hooij O, Jannink SA, Witjes JA, Verhaegh GW, et al.: Predictive value of PCA3 in urinary sediments in determining clinicopathological characteristics of prostate cancer. Prostate. 2010; 70: 10-6.

3. Papatsoris A, Anagnostopoulos F: Prostate cancer screening behaviour. Public Health. 2009; 123: 6971.

4. Schalken J: Interview with Jack Schalken. PCA3 and its use as a diagnostic test in prostate cancer. Interview by Christine McKillop. Eur Urol. 2006; 50: 153-4.

5. Hessels D, Schalken JA: The use of PCA3 in the diagnosis of prostate cancer. Nat Rev Urol. 2009; 6: 255-61.

6. Salagierski M, Verhaegh GW, Jannink SA, Smit FP, Hessels D, Schalken JA: Differential expression of PCA3 and its overlapping PRUNE2 transcript in prostate cancer. Prostate. 2010; 70: 70-8.

7. Bussemakers MJ, van Bokhoven A, Verhaegh GW, Smit FP, Karthaus HF, Schalken JA, et al.: DD3: a new prostate-specific gene, highly overexpressed in prostate cancer. Cancer Res. 1999; 59: 5975-9.

8. Marks LS, Bostwick DG: Prostate Cancer Specificity of PCA3 Gene Testing: Examples from Clinical Practice. Rev Urol. 2008; 10: 175-81.

9. Sokoll LJ, Ellis W, Lange P, Noteboom J, Elliott DJ, Deras IL, et al.: A multicenter evaluation of the PCA3 molecular urine test: pre-analytical effects, analytical performance, and diagnostic accuracy. Clin Chim Acta. 2008; 389: 1-6.

10. Tosoian JJ, Loeb S, Kettermann A, Landis P, Elliot DJ, Epstein JI, et al.: Accuracy of PCA3 measurement in predicting short-term biopsy progression in an active surveillance program. J Urol. 2010; 183: 534-8.

11. Vlaeminck-Guillem V, Ruffion A, André J, Devonec M, Paparel P: Urinary prostate cancer 3 test: toward the age of reason? Urology. 2010; 75: 447-53.

12. Kirby RS, Fitzpatrick JM, Irani J: Prostate cancer diagnosis in the new millennium: strengths and weaknesses of prostate-specific antigen and the discovery and clinical evaluation of prostate cancer gene 3 (PCA3). BJU Int. 2009; 103: 441-5.

13. Deras IL, Aubin SM, Blase A, Day JR, Koo S, Partin AW, et al.: PCA3: a molecular urine assay for predicting prostate biopsy outcome. J Urol. 2008; 179: 1587-92.
14. Chrisofos M, Papatsoris AG, Lazaris A, Deliveliotis C: Precursor lesions of prostate cancer. Crit Rev Clin Lab Sci. 2007; 44: 243-70.

15. Schilling D, de Reijke T, Tombal B, de la Taille A, Hennenlotter J, Stenzl A: The Prostate Cancer gene 3 assay: indications for use in clinical practice. BJU Int. 2010; 105: 452-5.

16. Chun FK, de la Taille A, van Poppel H, Marberger M, Stenzl A, Mulders PF, et al.: Prostate cancer gene 3 (PCA3): development and internal validation of a novel biopsy nomogram. Eur Urol. 2009; 56: 65967.

17. Whitman EJ, Groskopf J, Ali A, Chen Y, Blase A, Furusato B, et al.: PCA3 score before radical prostatectomy predicts extracapsular extension and tumor volume. J Urol. 2008; 180: 1975-8; discussion 1978-9.

18. Ankerst DP, Groskopf J, Day JR, Blase A, Rittenhouse $\mathrm{H}$, Pollock BH, et al.: Predicting prostate cancer risk through incorporation of prostate cancer gene 3. J Urol. 2008; 180: 1303-8; discussion 1308. Erratum in: J Urol. 2009; 181: 1507.

19. Haese A, de la Taille A, van Poppel H, Marberger M, Stenzl A, Mulders PF, et al.: Clinical utility of the PCA3 urine assay in European men scheduled for repeat biopsy. Eur Urol. 2008; 54: 1081-8.

20. Shappell SB, Fulmer J, Arguello D, Wright BS, Oppenheimer JR, Putzi MJ: PCA3 urine mRNA testing for prostate carcinoma: patterns of use by community urologists and assay performance in reference laboratory setting. Urology. 2009; 73: 363-8.

Accepted after revision:

March 4, 2010

\section{Correspondence address:}

Dr. Athanasios G. Papatsoris

University Department of Urology

Sismanoglio Hospital

Sismanogliou 1

Athens, 15126, Greece

E-mail: agpapatsoris@yahoo.gr 


\section{EDITORIAL COMMENT}

In the review article by Bourdoumis et al., the clinical utility of prostate cancer antigen 3 (PCA3) as a diagnostic marker for prostate cancer is highlighted. Currently, prostate cancer screening guidelines have failed to take a strong position on the merits of prostate cancer screening due in large part to the ongoing controversies on the survival benefit imparted from prostate cancer screening using a combination of digital rectal examination and serum prostate-specific antigen (PSA) level measurements (1). In consequence, clinicians have been seeking a better diagnostic and prognostic test for prostate cancer.

The preliminary studies evaluating the diagnostic utility of PCA3 would suggest that it may have great potential either as a substitute or adjunctive clinical tool for patients suspected of harboring prostate cancer and hence, requiring transrectal ultrasound guided prostatic biopsies. Prior to making more definitive recommendations on the role of PCA3 as a screening/diagnostic tool for prostate cancer, a multi-institutional prospective clinical trial comparing PSA and PCA3 will be required. Similarly, we are currently embarking in a new era of medicine in which a personalized approach to patient care can be adapted utilizing not only conventional clinical parameters but as well tumor cytoarchitectural and tissue expression patterns (2).
As clinicians, we are constantly seeking better diagnostic and therapeutic tools to help tailor treatment recommendations based on tumor aggressivity and risk of progression while taking into account treatment related morbidity and quality of life.

This discussion is particularly pertinent for prostate cancer as we clearly know that a significant proportion of patients have clinically insignificant disease and may be candidates for active surveillance. Clinical tools enabling us to identify patients at low risk of disease progression may change the treatment paradigm. In consequence, aggressive local therapy can therefore be offered to those best suited.

\section{REFERENCES}

1. Andriole GL, Crawford ED, Grubb RL 3rd, Buys SS, Chia D, Church TR, et al.: Mortality results from a randomized prostate-cancer screening trial. N Engl J Med. 2009; 360: 1310-9. Erratum in: N Engl J Med. 2009; 360: 1797.

2. Donovan MJ, Khan FM, Fernandez G, Mesa-Tejada R, Sapir M, Zubek VB, et al.: Personalized prediction of tumor response and cancer progression on prostate needle biopsy. J Urol. 2009; 182: 125-32.

Dr. Philippe E. Spiess

Division of Urology

H. Lee Moffitt Cancer Center

Tampa, Florida, USA

E-mail:Philippe.Spiess@moffitt.org 